\title{
Other Targetable Sarcomas
}

\author{
Veridiana Pires de Camargo, ${ }^{a, 1}$ Matt van de Rijn, ${ }^{b, 1}$ Enrique de Alava, ${ }^{c, 1}$ Juan Madoz-Gúrpide, ${ }^{c, 1}$ \\ Silvana Pilotti, ${ }^{d, 1}$ Margaret von Mehren, ${ }^{e, 1}$ Florence Pedeutour, ${ }^{f, 1}$ Robert G. Maki, ${ }^{a, 1}$ \\ Piotr Rutkowski, ${ }^{, 2}$ and David M. Thomas ${ }^{h, 2}$
}

\begin{abstract}
Despite complex genetics, aneuploid tumors like dedifferentiated liposarcoma have specific and reproducible chromosomal changes such as amplification of HDM2 and CDK4 that represent potential targets for systemic therapy. In addition, there are cancer cell survival pathways that may not be the target of chromosomal translocations or mutations that are still estimable targets for new systemic therapeutics, be it pathways involved in angiogenesis or apoptosis. In this review, we examine target selection for specific sarcoma subtypes, and demonstrate with a few examples new techniques being used to delineate novel therapeutic inroads for patients with sarcoma.

Semin Oncol 36:358-371 @ 2009 Elsevier Inc. All rights reserved.
\end{abstract}

\section{SELECTING A TREATMENT TARGET IN SARCOMA}

$\mathrm{O}$ ne of the primary tenets of oncology is to determine whether treatment is going to be curative or palliative in intent. This basic distinction has marked ramifications regarding the desir-

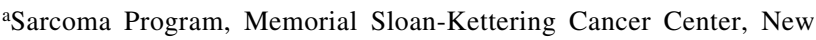
York, NY.

bDepartment of Pathology, Stanford University Medical Center, Stanford, CA.

cMolecular Pathology Program, Centro de Investigación del CáncerIBMCC, University of Salamanca-CSIC, Salamanca, Spain.

dLaboratory of Experimental Molecular Pathology, Department of Pathology, Fondazione IRCCS, Istituto Nazionale Tumori, Milan, Italy.

eSarcoma Oncology, Fox Chase Cancer Center, Philadelphia, PA.

fLaboratoire de Génétique des Tumeurs Solides, Faculté de Médecine, Centre Hospitalier Universitaire de Nice et CNRS UMRS 6543, Nice, France.

'Maria Sklodowska-Curie Memorial Cancer Center and Institute of Oncology, Department of Soft Tissue/Bone Sarcoma and Melanoma, Warsaw, Poland.

hesearch Division, Peter MacCallum Cancer Centre, East Melbourne, Victoria, Australia.

1,2 These authors contributed equally to this work.

Enrique de Alava is supported by the European Commission ( $\mathrm{NoE}$ EuroBoNet), Ministry of Science and Innovation of Spain-FEDER (PI052524, RD06/0020/0059). Silvana Pilotti is supported by Associazione Italiana per la Ricerca sul Cancro (AIRC). Robert G. Maki is supported by Program Project Grant No. P01-CA47179; Cycle for Survival. David M. Thomas is supported by the Victorian Cancer Agency Clinician Researcher fellowship, and Cancer Australia.

Address correspondence to David M. Thomas, FRACP, PhD, Research Division, Peter MacCallum Cancer Centre, St Andrew's Place, East Melbourne, Victoria 3002, Australia. E-mail: david.thomas@ petermac.org

0270-9295/09/\$ - see front matter

(C) 2009 Elsevier Inc. All rights reserved.

doi:10.1053/j.seminoncol.2009.06.008 ability of local and systemic therapeutic options alike. While surgery, radiation, and standard cytotoxic chemotherapy are frequently employed in both primary and metastatic therapy for patients with sarcomas, these modalities are neither tumor-specific nor tumor subtype-specific. Particularly for cytotoxic chemotherapy, there is typically limited benefit, except in the case of adjuvant therapy of pediatric sarcomas, in which case chemotherapy is mandatory given its high rate of efficacy in primary disease.

Is it possible to think about metastatic disease as ultimately curable? As ever is the case for sarcomas, gastrointestinal stromal tumor (GIST) provides a paradigm. Prior to 2000, recurrent GIST was uniformly fatal, given the overall resistance of GIST to standard cytotoxic chemotherapy. While biological factors such as elevated multidrug resistance proteins may explain some of this effect, there are likely other factors that we still do not understand as the principal reasons for overt chemotherapy resistance of GIST

The identification of mutations in KIT in GIST $^{1} \mathrm{com}-$ pletely transformed the therapy of GIST with imatinib and other tyrosine kinase inhibitors commercially approved or under study at present. We also understand that there are variations in the risk of recurrence of GIST based on mitotic rate, size, and anatomical location of the primary tumor. These findings are underscored by the finding of unique gene expression array patterns of GIST based on anatomical location, which have begun to have an impact on patient care with the finding in one study of the importance of higher dose imatinib in improving progression-free survival (PFS) on this agent. However, overall survival is no different based on dose, probably due to effects of crossing over of patients from lower to higher dose imatinib. 
Table 1. Lessons on Selecting a Drug to Test Against Sarcoma (or other cancer)

1. The target must be biologically relevant, eg, KIT and PDGFR in GIST

2. Treatment need not be cytotoxic for benefit, though such benefit becomes more difficult to evaluate in at least some situations

3. Therapy must have limited toxicity due to the need for chronic administration

4. Over time, cancer cells evolve mechanisms to bypass the inhibited target

Thus, GIST has taught us important lessons on how to match a specific drug to a specific sarcoma (or other cancer) subtype (Table 1). Is the presence of a target sufficient? Clearly not. In the setting of small cell lung cancer and seminoma, there is abundant KIT expression, yet the response rate to imatinib in these diseases is essentially zero. For an example with sarcomas, in the case of malignant peripheral nerve sheath tumor and synovial sarcoma, there is both expression and amplification of HER1/EGFR; however, clinical trials of epidermal growth factor receptor (EGFR)-targeted therapy yielded a median PFS of 2 months, ie, one scanning interval.

The striking results with trabectedin (ET-743) in myxoid/round cell liposarcoma (M/RCLS) ${ }^{2}$ also highlight the use of a novel agent in a specific sarcoma subtype. Trabectedin is both a minor groove alkylator, like mitomycin, but also interferes with transcriptioncoupled nucleotide excision repair and inhibits the transcription of heat shock-inducible genes, causing DNA strand breaks, late S/G2 cell cycle arrest, and p53-independent apoptosis. M/RCLS, the second most common class of liposarcomas, are particularly sensitive to trabectedin for unclear reasons. Despite its well recognized $\mathrm{t}(12 ; 16)$ involving genes FUS-CHOP, FUS$C H O P$ fusion gene expression did not change in quantity before and after therapy, leaving some question as to mechanisms of action for a given patient's tumor. ${ }^{2}$ Other factors may be involved in this pattern of sensitivity, as borne out by the BRCA1/ERCC1 fingerprint of sarcomas found to be sensitive and insensitive to trabectedin. ${ }^{3}$

How do we know when a target is relevant? Through an understanding of tumor pathophysiology, understanding what and how the drug blocks the target, and identification of the ideal patient population for each treatment. While for GIST this was relatively straight forward, it is much less clear for other targeted agents, given the lower frequency of specific mutations in critical genes identified in other sarcomas to date. There are ongoing efforts from bench to translational researcher to bedside, to synthesize data from genomic and proteomic studies, to evaluate the biological implications of findings, to determine how possible it is to block a target, and to develop the strategies to test new agent most effectively, not to mention trying to identify emerging resistance mechanisms.

Given the present state of available agents and what has been seen to date with single agents, it is clear the cure rate for patients with metastatic disease will probably only increase with the use of combinations of targeted therapeutics (and perhaps also with standard cytotoxic drugs). If we cannot achieve cure for the time being, hopefully it is possible for other sarcomas, like GIST, to make them truly chronic diseases and not immediately life-threatening ones.

\section{GENE PROFILING TO FIND TARGETS}

We have been the middle of an explosion of information regarding patterns of gene and protein expression of sarcomas. It is clear that even small amounts of data regarding specific mRNA expression markers in specific sarcoma subtypes can be rapidly confirmed on larger scale tissue microarrays using immunohistochemistry and in situ hybridization. These types of studies have led to identification of markers such as DOG1 for GIST, ${ }^{4-6}$ TLE1 in synovial sarcoma,${ }^{7}$ S100P in bladder carcinoma, ${ }^{8-10}$ and CK17 in breast adenocarcinoma. ${ }^{11}$ With the development of new diagnostic and prognostic markers, we can recognize subsets of cases within a diagnostic entity, and thus more accurately target novel therapeutics as they become available.

We see from the examples of GIST (KIT and PDGFRalpha) that high expression of a tyrosine kinase receptor (TKR) is associated with a mutation in the protein itself, or sometimes a translocation involving the ligand for that receptor, eg, PDGFR-beta in dermatofibrosarcoma protuberans (DFSP).

Matt van de Rijn and colleagues hypothesized that screening for high expression of TKRs on a tissue microarray would identify novel targets for sarcoma therapeutics. In this way CSF1 ligand was identified in a small portion of cells in tenosynovial giant cell tumor (TGCT) and pigmented villonodular synovitis (PVNS), while nearly all cells in the tumor are positive for the receptor, CSF1R. ${ }^{12} \mathrm{~A}$ translocation was demonstrated involving chromosomes 1 and 2 using breakaway fluorescence in situ hybridization (FISH) probes, but the investigators found this translocation only in a minority of the tumor cells, indicating that the tumor cells expressed CSF1 and recruited inflammatory cells such as monocytes into the lesion, consistent with the inflammatory appearance of such lesions. Like DFSP, this leads to an autocrine loop as well, which drives tumor proliferation and survival. Remarkably, a recent case report indicates that imatinib, which blocks not only platelet-derived growth factor (PDGF) receptors, KIT, 
and BCR-ABL but also CSF1R, can lead to clinical responses in patients with TGCT/PVNS. ${ }^{13}$

These data have now been used to model a different type of cancer and its outcome. High plasma levels of CSF1 expression are associated with breast cancer risk. ${ }^{14}$ Furthermore, in animal models a decrease in tumor-associated macrophages through decreasing CSF1 levels inhibits tumor progression. ${ }^{15}$ It was thus hypothesized that expression profiling of TGCT/PVNS would define a CSF1-based gene expression profile. A 360-gene pattern typical of TGCT/PVNS was defined, and then used to cluster 295 breast carcinomas by this pattern. This gave two clusters of tumors, some with the TGCT/PVNS CSF1 gene signature, and others without. This gene set was filtered through five different breast cancer datasets to further narrow the key genes of the CSF1 gene signature, and showed that this gene set is associated with a higher grade of tumor and negative estrogen receptor expression. ${ }^{16-18}$

This gene profile also has been applied to leiomyosarcoma (LMS). We knew that CD163/CD68 histiocyte numbers correlated with poor outcome in extrauterine (but not uterine) $\mathrm{LMS}^{19}$. Both uterine and nonuterine LMS could be clustered with this basis set of CSF1related genes, and those people with a CSF1 expression pattern in their LMS fared worse than those without the CSF1 expression pattern. ${ }^{19}$ Since CSF1 could be shown to be produced by at least some LMS cells, CSF1 and its downstream targets thus become a target for therapy for LMS, which will have to be evaluated prospectively in trials of imatinib in patients with tumors with this expression pattern.

\section{THE ROLE OF PROTEOMICS \\ IN THE SEARCH FOR NEW THERAPEUTIC TARGETS IN SARCOMAS}

\section{Relevance of Proteomics in the Discovery of New Therapeutic Targets in Sarcomas}

Frequently, many relevant discoveries in biomedical sciences have their origins in the investigation of differences between one functional state of a biological system versus another. Differences between cellular states are reflected in changes in gene expression that manifest themselves at the level of both the messenger (mRNA) and the final product (protein). Proteins are almost always the effectors of biological functions, but protein levels depend not only on the levels of the corresponding messages but also on a multitude of translational controls, post-translational modifications, and regulated degradation. ${ }^{20,21}$ The expression levels of all proteins would arguably provide the most relevant single data set characterizing a biological system.

A number of decisive breakthroughs in proteomics have materialized, including protocols to handle small amounts of biological samples, the ability to rapidly identify peptides, and the direct analysis of very complex protein mixtures. ${ }^{22,23}$ Over the last two decades, two-dimensional electrophoresis (2-DE) has established itself as the de facto approach to separating proteins from cell and tissue samples. More recently, mass spectrometry (MS) also has become a powerful tool for detecting posttranslational modifications and protein interactions. Even now, progress on quantitative methods has allowed the determination of quantitative systems-wide measurement of protein expression levels. ${ }^{24}$ Nowadays, the conceptual strategies and technological achievements of "proteomics," which aims to provide just that, have proven ready to tackle comprehensive protein expression analysis, even at a systems-wide level. $^{25}$ The combination of complementary proteomics tools, such as 2-DE, matrix-assisted laser desorption/ ionization-time of flight mass spectrometry (MALDI-ToF MS), and bioinformatics offer to the biomedical researcher an opportunity to study the profile of changes in protein levels.

In many cases, sarcoma cell lines are employed as model systems wherein mechanistic molecular schemes are studied. Control of cell-cycle progression, mediation of invasion and migration, evasion of apoptosis, etc constitute checkpoints that are usually investigated in cell line models of different types of tumors in response to different agents. Using different models of sarcoma xenografts (SK-N-MC and IMR32 [neuroblastoma], RH1 and RH30 [rhabdomyosarcoma], and KHOS/NP [osteosarcoma]), Izbicka et al evaluated and compared the effects of docetaxel and paclitaxel. ${ }^{26}$ The approach used immunoblotting and surface-enhanced laser desorption/ionization (SELDI) MS to assess drug effects on the expression of the beta-tubulin isotypes and apoptotic markers (Bcl-2, Bax, Bcl-XL). However, the results of this anticancer activity showed no apparent correlation with drug effects on those proteins. The drugs had significantly different, yet highly heterogeneous effects on the tumor levels of the proteins. In contrast, six protein species identified by proteomic profiling were consistently and differentially regulated by docetaxel and paclitaxel in all xenografts. ${ }^{26}$

The study of Ewing sarcoma also has provided examples of how proteomics may deepen our understanding about the mechanisms regulating the response to different treatments. Ewing sarcoma expresses several deregulated autocrine loops mediating cell survival and proliferation that contribute to its pathogenesis. Insulin-like growth factor 1 receptor (IGF1R) and KIT are transmembrane receptors that mediate two of these loops, ${ }^{27,28}$ and are therefore directly involved in the growth and survival properties of Ewing sarcoma. ${ }^{29-31}$ Their blockade, therefore, is a promising therapeutic approach for this neoplasm. Martins et al reported the in vitro impact of IGF1R/KIT pathway blockade on Ewing sarcoma cell lines, ${ }^{32}$ and they have extended 
their observations to the level of proteomic changes induced by this treatment, to find and validate new possible therapeutic targets. ${ }^{33}$ Two-dimensional gel electrophoretic analysis and MALDI-ToF studies of Ewing sarcoma cell lines treated with ADW742 and/or imatinib (specific IGF1R/KIT inhibitors) revealed a large panel of differentially expressed proteins, some of them related to stress-induced response. Among them, the changes in protein expression between cell lines sensitive and resistant to IGF1R/KIT inhibitors were particularly significant in the case of heat shock protein 90 (HSP90). ${ }^{33}$

Response to stress is a key mechanism conditioning drug sensitivity. Stress-protective HSPs are often overexpressed in neoplastic tissues and cancer cell lines, and therefore HSP inhibition might become a new therapeutic strategy to inhibit multiple receptor pathways. The authors illustrated that inhibition of HSP9O with 17-AAG (a specific HSP90 inhibitor) and siRNA against HSP90 reduced Ewing sarcoma cell line growth and survival, especially in cell lines that previously showed resistance to IGF1R/KIT pathway blockade. ${ }^{33}$ 17-AAG treatment induced HSP90 client protein degradation, including AKT, KIT, or IGF1R, by inhibiting their physical interaction with HSP90. Animal models confirmed that HSP90 inhibition, alone or combined with IGF1R inhibition, significantly reduced tumor growth and expression of client proteins. The authors postulate, for the first time in Ewing sarcoma, that in addition to the levels of expression and basal activation of IGF1R/KIT, the development level of the stress response mechanism is another important determinant of sensitivity to IGF1R inhibitors or KIT inhibitors in Ewing sarcoma cell lines. Importantly, targeting HSP90 function might be of therapeutic value in Ewing sarcoma, especially in cases of previous resistance to KIT or IGF1R pathway blockade.

\section{Challenges and Near-Term Perspectives for Proteomics in Sarcoma Research}

We are convinced that, along with cellular, molecular, and genomic advances, novel proteomic approaches may be more efficient and allow monitoring of changes in protein expression, modification, and differentiation patterns associated with fusion protein expression in sarcomas. It also has been suggested that yet unidentified genetic factors cooperate with the chimeric transcription factors to induce oncogenesis. Proteomics also should allow the delineation of the downstream pathway(s) unleashed by the aberrant malfunction of sarcoma chimeric proteins. All of the genes and proteins involved in a game of consecutive, orchestrated, anomalous interactions should be unraveled, so the effectors of endpoints of chimera expression in different cellular contexts could be targeted. Even more subtle qualitative and quantitative differences in expression patterns are associated with different sarcoma fusion types that result from variability in genomic breakpoint locations. Bearing in mind that the majority of molecular effectors in cellular processes are proteins, and that most new anti-cancer agents currently available are addressed against protein targets, we believe that a proteomic perspective, complementary to that of genomic biology, would bring extended choices to render new therapies in sarcoma treatment.

Sarcoma chimeric proteins with DNA binding domains may induce tumorigenesis by perturbing gene expression. At the same time, they also interact with other proteins involved in different aspects of mRNA and DNA metabolism, suggesting novel physiological roles in the global process of tumor formation. A comprehensive characterization of the molecular structure of the fusion protein would provide greater detail of the mechanisms underlying the functional activities of the chimeras. In return, all of this knowledge will provide clues to allow us to develop sarcoma proteinspecific inhibitors with therapeutic potential. Given the unique specific interactions of every sarcoma protein with other cellular proteins ${ }^{34}$ and the absolute tumor specificity of fusion proteins, this possibility may well come to pass in the near future.

Finally, we also must be cognizant of the potential of research in posttranslational protein modifications (PTMs). PTMs are covalent processing events that change the properties of a protein by proteolytic cleavage or by addition of a modifying group to one or more amino acids. They may alter physical and chemical properties, folding, conformation distribution, stability, activity, and, consequently, function of the proteins. PTMs modulate the activity of most eukaryote proteins, and can determine its activity state, localization, turnover, and interactions with other proteins. Examples of the biological effects of protein modifications include phosphorylation for signal transduction, ubiquitination for proteolysis, attachment of fatty acids for membrane anchoring and association, glycosylation for protein half-life, targeting, and cell-cell and cell-matrix interactions. In signaling, for example, kinase cascades are turned on and off by the reversible addition and removal of phosphate groups. ${ }^{35}$ Consequently, the analysis of proteins and their posttranslational modifications is critical for the study of cancer, and we envision that this might be particularly the case for sarcomas, where the function of the chimera protein determines the cascade of events ultimately leading to tumorigenesis.

Despite advances in our understanding of the biology of sarcomas, more remains to be learned about the factors that impart varying clinical outcomes in this disease. This is necessary to further expand therapeutic options and identify better prognostic factors that would make a difference in patient outcomes. 


\section{NON-MUTATIONAL RECEPTOR TYROSINE KINASE ALTERATIONS IN SARCOMAS}

Sarcomas carrying non-mutational receptor tyrosine kinase (RTK) alterations are a subset of sarcomas that are addicted through an RTK activation mechanism (represented by an autocrine/paracrine loop or the gain or loss of a gene or chimeric fusion protein) that can be targeted by selective drugs. While in general the presence of target mutations has predicted responses to molecular therapeutic agents, in some cases the activation of druggable RTK pathways is not associated with mutational events. In addition, even where mutations have been defined, the druggable target may represent a downstream mediator of this RTK pathway, rather than the mutant itself. These "non-mutational" alterations are of substantial clinical interest, since within any given cancer, mutations may affect different elements of multi-component pathways. The most distal, obligate downstream component may be the most rational target for the tumor class as a whole, regardless

\begin{tabular}{|c|c|}
\hline Patient A & Activated receptors \\
\hline Highest & EGFR \\
\hline$\underset{\underline{\Phi}}{\bar{\Phi}}$ & PDGFRB \\
\hline $\bar{c} \bar{c}$ & Her2/neu \\
\hline $\begin{array}{c}\mathscr{S} \\
\Phi\end{array}$ & Her4 \\
\hline ய̊ & Flt3 \\
\hline Lowest & MCSFR \\
\hline
\end{tabular}

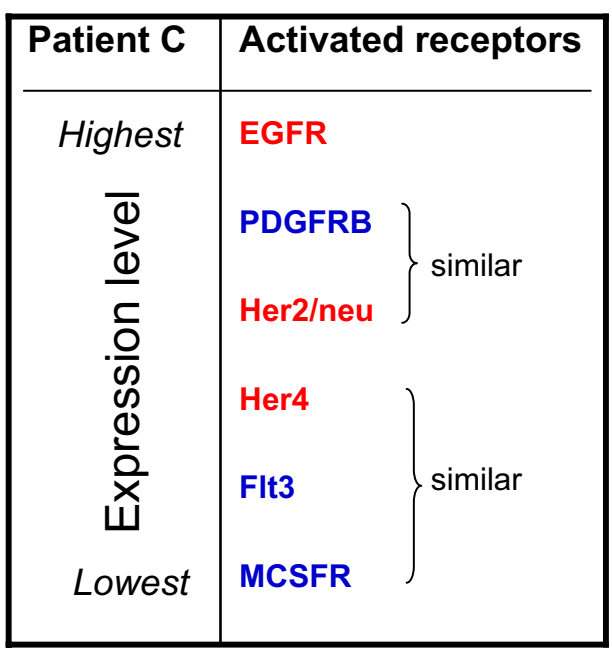

of mutation status, rather than using different drugs for different specific mutations.

The challenge is to identify these key RTK pathways. Over the last few years, a number of reports have described possibly targetable profiles in various several sarcomas, ${ }^{36-47}$ but fewer than half of these tumors showed clinical responses. ${ }^{37,41,43,46-48}$ We will here concentrate on two responding sarcomas: chordomas and alveolar soft part sarcomas (ASPS), and give real examples of detection of activation of kinases in these tumors as a means how this type of research can inform clinical studies of new agents.

\section{Chordoma}

PDGFR-beta appears to be activated by an autocrine loop in chordomas; targeting this receptor by means of a selective tyrosine kinase (TK) inhibitor (imatinib) leads to therapeutic benefits and objective imaging responses. ${ }^{41,42,48,49}$ However, the observation of primary resistance to imatinib in some patients prompted us to

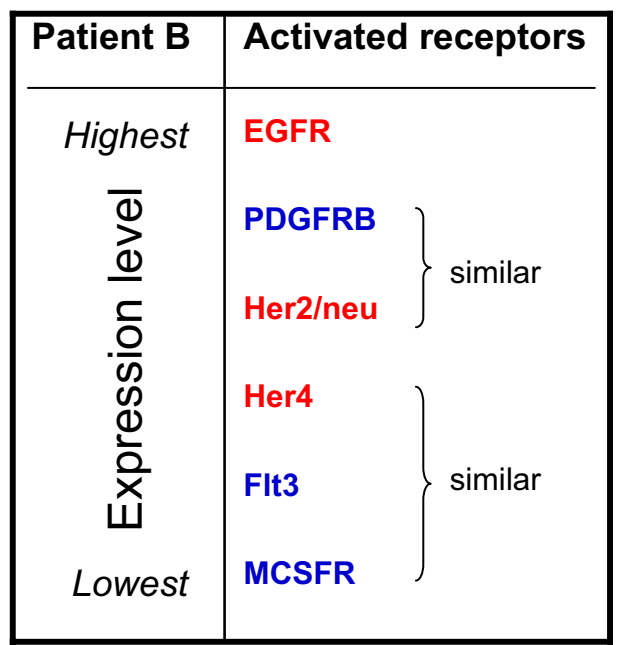

\begin{tabular}{|c|c|}
\hline Patient D & Activated receptors \\
\hline Highest & EGFR \\
\hline$\underset{\Phi}{\Phi}$ & Her2/neu \\
\hline$\frac{}{ㅇ}$ & MCSFR \\
\hline d) & PDGFRB \\
\hline ய் & Flt3 \\
\hline Lowest & No Her4 \\
\hline
\end{tabular}

Figure 1. Phosphokinase dot blot for four patients with chordoma. Activation of PDGFR-beta, EGFR, and HER2 is evident in most cases. 83 
reanalyze our cryopreserved case material from the preimatinib era for the presence of other activated RTKs.

Using human phospho-RTK antibody arrays, upstream targets in four randomly selected cases were examined, and surprisingly, in addition to the recognized activation of imatinib-sensitive PDGFR-beta and RTKs of the same family (Flt3 and MCSFR1), activation of RTKs belonging to the EGFR family were observed: mainly EGFR itself (HER1), followed by HER2/neu and, to a lesser extent, HER4 (Figure 1).

These data were confirmed by biochemical analyses of the same specimens and, interestingly, comparison of the biochemical and immunohistochemical data showed that all but one of the cases tested for EGFR were negative for EGFR and Her2/neu protein expression, whereas comparison of the biochemical and FISH data of all of the cases showed that both genes were deregulated, and with an identical profile, thus suggesting that FISH may be a more useful predictive assay than immunohistochemistry.

Subsequent analysis of the downstream targets showed that both the PI3K/AKT and RAS/ERK 1-2 pathways were activated in the absence of any mutations of the genes belonging to them (PI3KP110 and PTEN, and $R A S$ and $B R A F$ ). FISH analysis of $\mathrm{PI} 3 \mathrm{~K}$ did not reveal any numerical alterations, but more than half of the cases showed PTEN monosomy, which, however, seemed to have no effect on PTEN function as immu- noprecipitation experiments did not reveal any change in the amount of protein. Furthermore, in line with published data indicating that mammalian target of rapamycin (mTOR) is stimulated by both the RAS and PI3K pathways, ${ }^{50}$ we found that the mTOR complex 1 (raptor) and its target ribosomal protein $\mathrm{S6}$ were expressed and activated, although the latter was only expressed and activated at a low level and, unexpectedly, not at all in two cases (Figure 2). One possible reason for the lack of 56 expression could be the loss of the 9p21 locus to which both S6 and p16 map: p16 was deregulated in $70 \%$ of the cases series and was homozygously deleted in the two cases not expressing S6.

These new insights into the TK profile of chordomas indicate that, in addition to or instead of PDGFR inhibitors, it may be useful to use inhibitors of EGFR, inhibitors of both EGFR (HER1) and HER2/neu, as well as inhibitors of mTOR/raptor.

\section{Alveolar Soft Part Sarcomas}

ASPS are rare tumors that mainly affect young patients and are clinically characterized by prolonged survival combined with a high rate of metastatic disease and chemo-resistance, and molecularly characterized by an ASPLCR1-TFE3 translocation. There is one published report with a case of ASPS with brain metastases successfully treated with angiogenic inhibitors, ${ }^{51}$

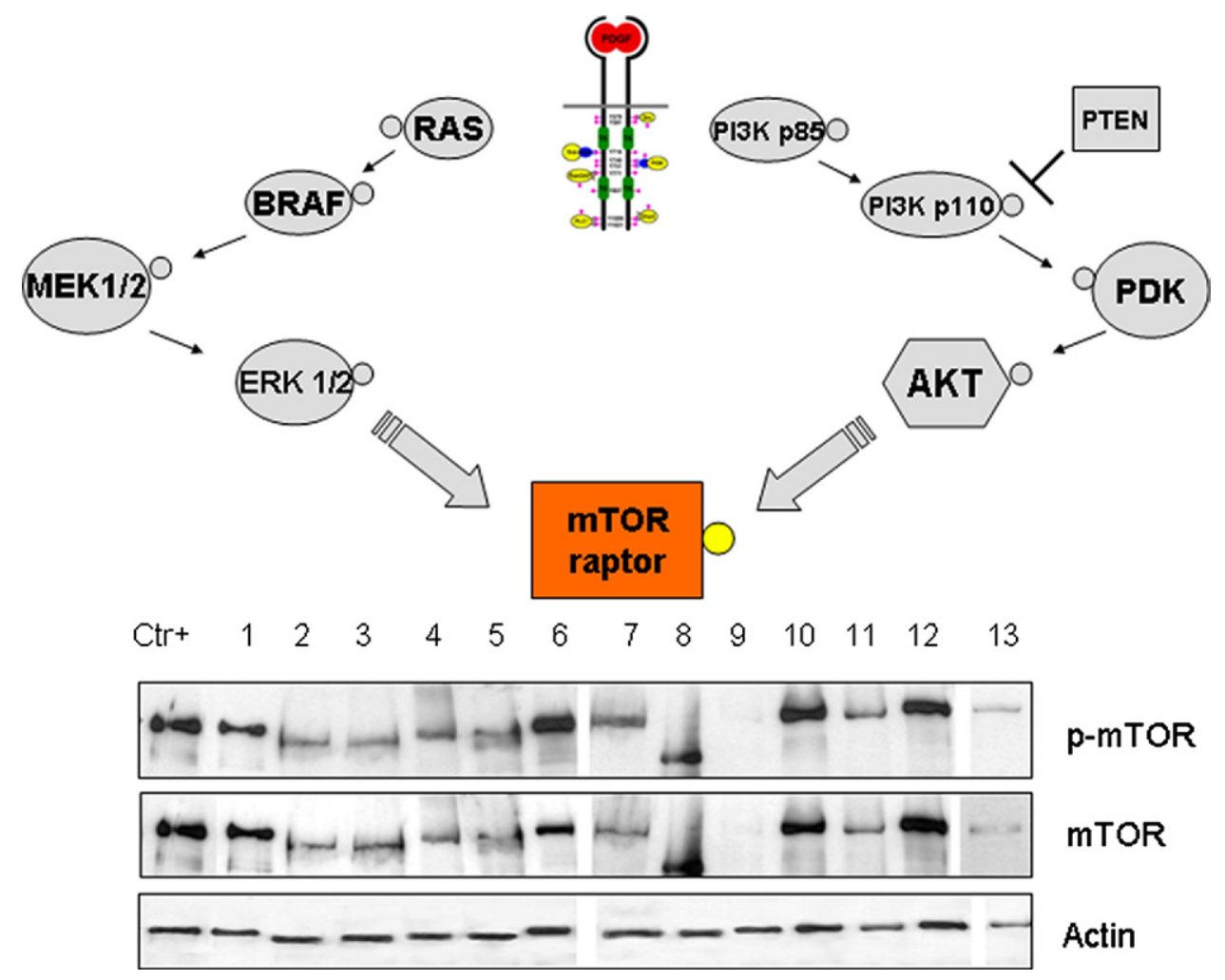

Figure 2. Western blot for mTOR1 and phospho-mTOR1 (raptor) in chordoma patient samples, showing activation of mTOR in most cases. (Courtesy of S. Pilotti.) 


\begin{tabular}{|c|c|c|c|}
\hline Patient A & Activated receptors & Patient B & Activated receptors \\
\hline 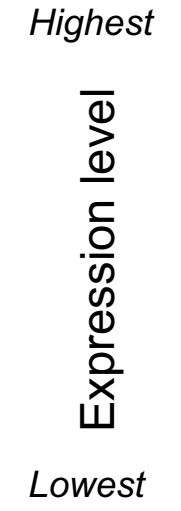 & $\begin{array}{l}\text { EGFR } \\
\text { PDGFRB } \\
\text { HGF and MSPR } \\
\text { FIt3 and MCSFR } \\
\text { Her4 } \\
\text { AxI and Dtk }\end{array}$ & 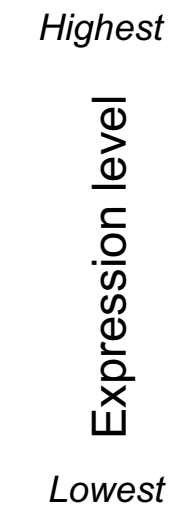 & $\left.\begin{array}{l}\text { PDGFRB } \\
\text { EGFR } \\
\text { FGFR3 }\end{array}\right\}$ similar \\
\hline Patient C & Activated receptors & Patient D & Activated receptors \\
\hline 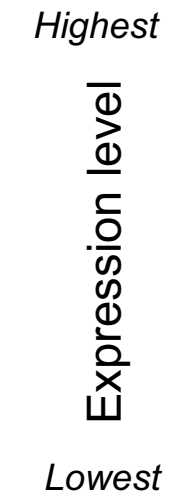 & $\begin{array}{l}\text { EGFR } \\
\text { PDGFRB } \\
\text { MSPR } \\
\text { FIt3 and MCSFR } \\
\text { Her4 } \\
\text { Axl and Dtk }\end{array}$ & 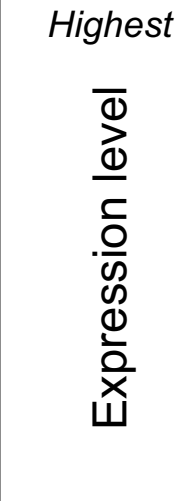 & 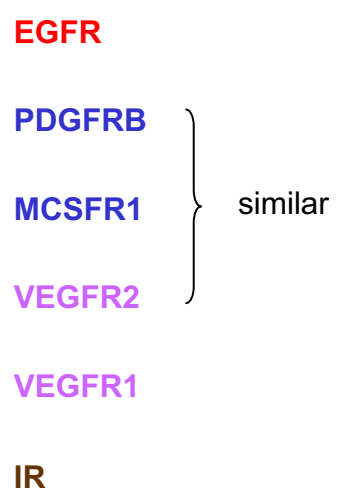 \\
\hline & & Lowest & Axl and Dtk \\
\hline
\end{tabular}

Figure 3. Phosphokinase dot blot for four patients with alveolar soft part sarcoma (ASPS). Activation of EGFR and PDGFRB is evident in most cases, with variable expression of other kinases as indicated. ${ }^{83}$

and two more recent papers have provided evidence that unregulated TFE3 overexpression in the context of the ASPLCR1-TFE3 fusion transcript plays a role in the pathobiology of ASPS.52,53

In the light of this finding, and reports of the clinical effectiveness of sunitinib (in two patients with disease regression) in stage IV ASPS patients, the RTK activation profile of four primary tumors was examined by investigating upstream and downstream targets, and the possible mechanisms of activation of the upstream RTKs using biochemical analysis of frozen material (using human phospho-RTK antibody arrays and IP/WB, and mutational and reverse transcriptase-polymerase chain reaction [RT-PCR] expression analyses) and immunohistochemistry and FISH analyses of related formalin-fixed, paraffin-embedded samples.

The upstream target analysis (RTK array and immunoprecipitation/Western blot) indicated an activation profile involving the PDGFR (PDGFR-beta, Flt3, or M-
CSFR), EGFR, and MET families in all four cases, and VEGFR1/VEGFR2 in one (Figures 3 and 4). We therefore investigated the downstream PI3K/AKT and RAS/ ERK pathways, which showed strong activation (as previously reported), ${ }^{53}$ as well as mTOR and its targets S6K and S6, which were all activated in the absence of any deregulation of upstream or downstream effectors (Figure 5). As this suggested the support of upstream RTK activation, we looked for the activation mechanisms by means of mutational and FISH analyses together with RT-PCR of the cognate ligands of PDGFR, EGFR, and MET, the results of which were consistent with the presence of ligand-dependent activation in all cases.

Taken together, these promising preliminary findings suggest that ASPS are mainly characterized by the autocrine/paracrine activation of PDGFRs, MET, and, to a lesser extent, VEGFR. The fact that all of these are targets of sunitinib provides a rationale for its use, but the ASPS TRK activation profile (supported by the ex- 


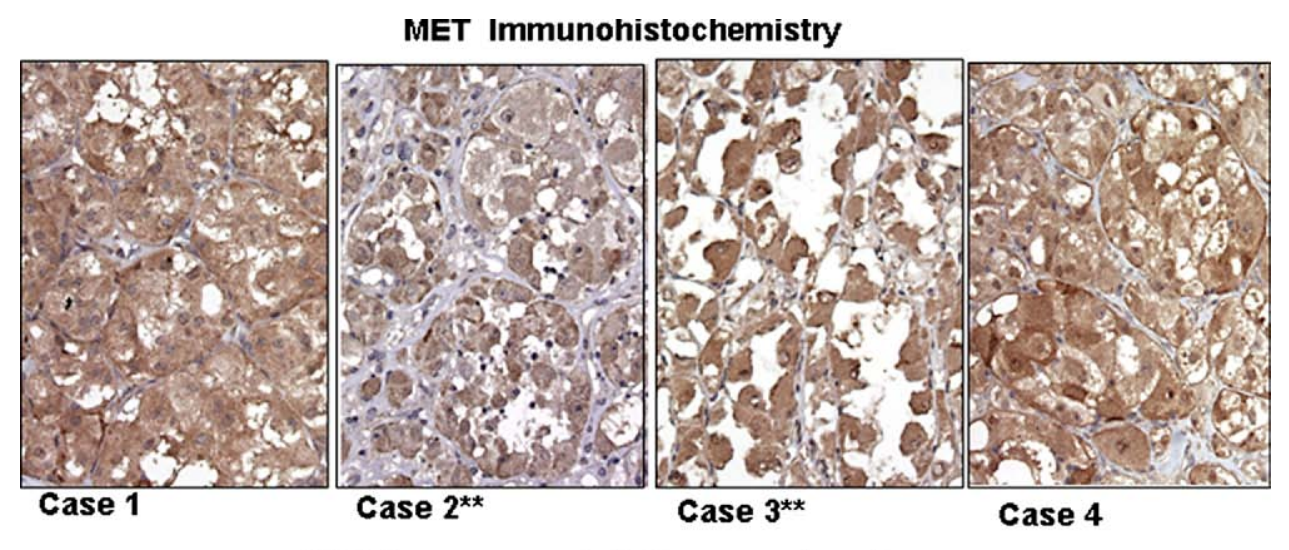

MET Immunoprecipitation experiments

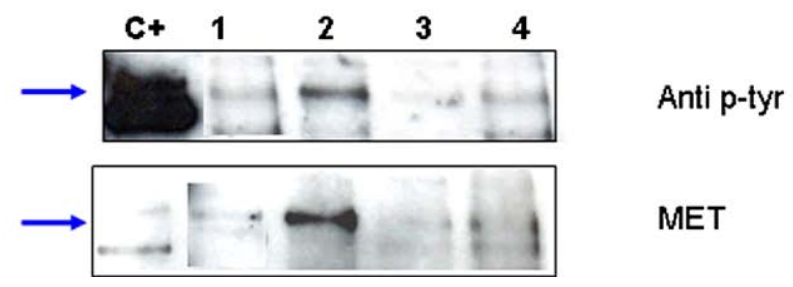

Figure 4. Robust expression of MET in ASPS patient samples, with evidence of phosphorylation (activation) of MET in at least some cases. (Courtesy of S. Pilotti.)

perimental data) also suggests that MET inhibitors may be useful. Furthermore, tumoral "addiction" to EGFR or other RTKs has to be ruled out in the case of sunitinibresistant tumors. Finally, these types of experiments will be very helpful for identifying new targets in other sarcoma subtypes.

\section{GENETICS OF WELL-DIFFERENTIATED/ DEDIFFERENTIATED LIPOSARCOMA IDENTIFYING TARGETS FOR THERAPY}

Well-differentiated and dedifferentiated liposarcomas (WD/DDLS) form a spectrum of tumors, and are the most common of the three forms of liposarcoma, representing about half of liposarcomas. M/RCLS

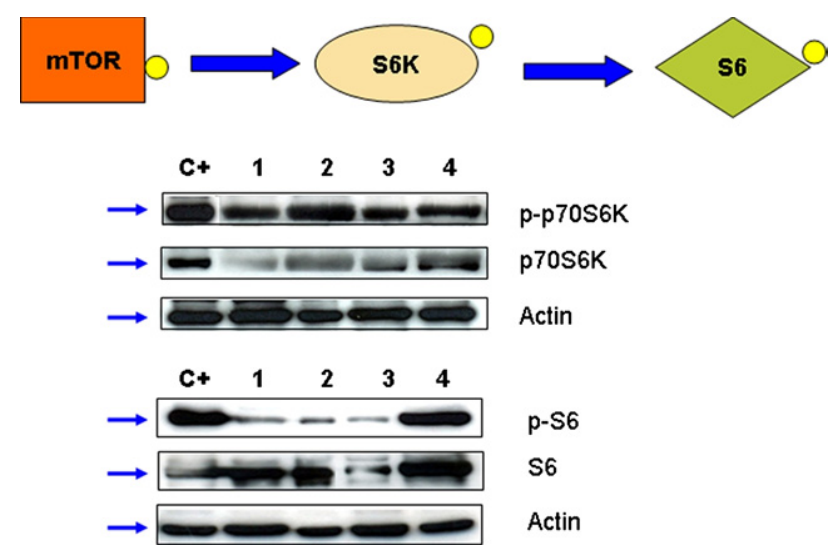

Figure 5. Activation of $\mathbf{S 6}$ kinase by Western blot in ASPS patient samples. (Courtesy of S. Pilotti.) (characterized by the $\mathrm{t}(12 ; 16)$ FUS-CHOP translocation) and the much less common pleomorphic liposarcoma (with an aneuploid karyotype more like high-grade malignant fibrous histiocytoma/high-grade differentiated pleomorphic sarcoma [MFH/HGUPS]) represent the other half of the liposarcomas observed. Lipomas, which also have some of the similar genetic alterations as WDLS, are at least 100 times more common as liposarcomas.

WDLS have a relatively simple karyotype that is largely diploid with supernumerary ring or giant marker chromosomes. As tumors are observed to be less histologically differentiated (DDLS), there is an increase in the complexity of the genotype, with polyploidy and aneuploidy common, as well as continued development of the ring and marker chromosomes, consistent with a spectrum of tumors with increasingly chaotic chromosomal arrangements as the tumor is found to be less differentiated.

The ring, giant, and marker chromosomes found in WD/DDLS are observed to contain amplifications of the chromosomal region $12 \mathrm{q} 14-15$ region that contains important oncogenes such as MDM2 and $C D K 4$, easily observed by FISH or by array comparative genomic hybridization $(\mathrm{CGH})^{54}$ (Figure 6). Interestingly, in terms of propagation of the aneuploid karyotype after cell division, ring, satellite, and marker chromosomes do not contain the characteristic alpha-satellite sequences of centromeric DNA as sites of spindle attachment during cell division, and are termed neocentromeres. 


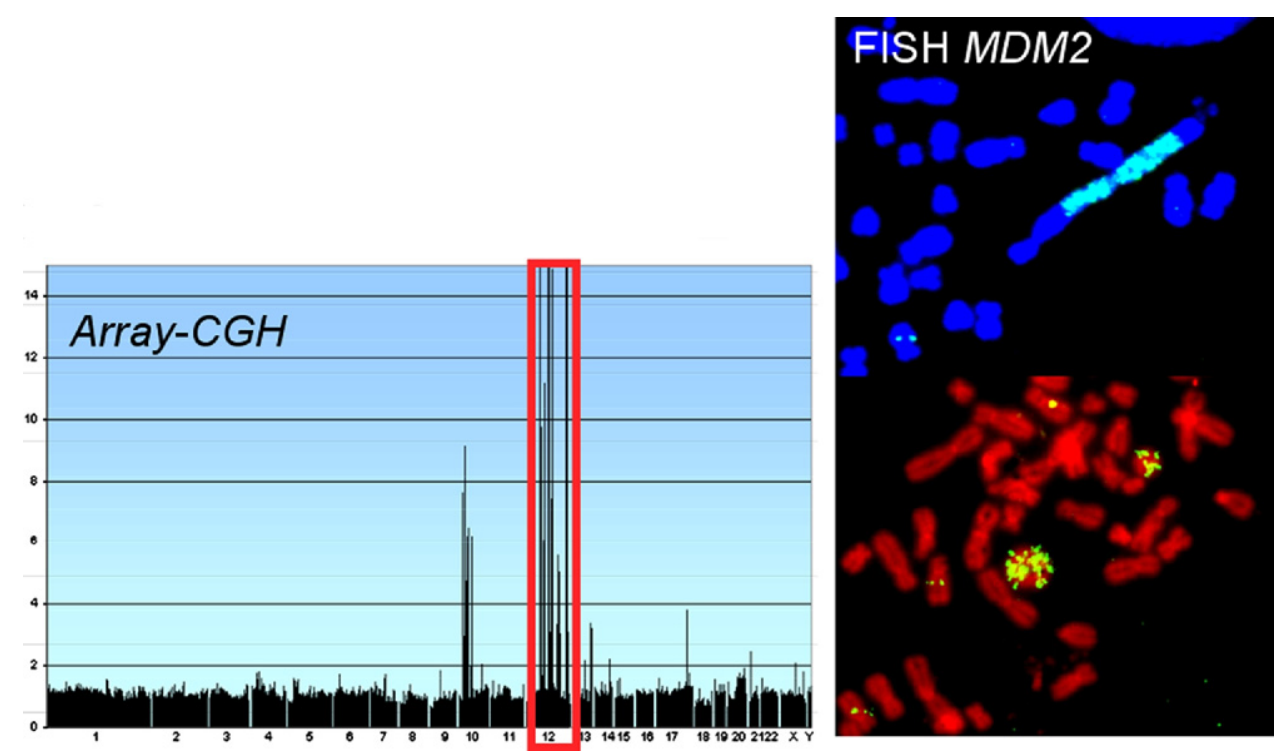

Figure 6. Amplification of MDM2 demonstrated in well-differentiated-dedifferentiated liposarcomas by a (left) array comparative genomic hybridization (CGH) and (right) fluorescence in situ hybridization (FISH). (Courtesy of A. Aurias and F. Pedeutour.)

MDM2 is found at $12 \mathrm{q} 15$, and $C D K 4$ at $12 \mathrm{q} 14.1$. The amplification of this region helps in distinguishing WDLS from lipomas, and DDLS from other poorly differentiated malignancies. ${ }^{55-58}$ Immunohistochemistry for MDM2 can be somewhat useful in diagnosis, but there are false positives, false negatives, and difficulties in interpretation of even technically well-executed immunohistochemistry. Molecular detection of MDM2 by FISH or quantitative polymerase chain reaction (QPCR) testing provides a better diagnostic for MDM2 amplification in these tumors. Tumors that are negative for amplification can be evaluated for the presence of other markers more characteristic of lipomas, M/RCLS, or other diagnoses. Short of wholesale amplification of chromosome 12q, low-level gains of MDM2 and/or CDK4 are observed in both lipomas and atypical lipomatous tumors (the lowest grade version of liposarcoma), perhaps representing the missing transition state between lipoma and overt WD/DDLS.

MDM 2 and $C D K 4$ are not the only genes amplified on the chromosome $12 \mathrm{q}$ amplicon of WD/DDLS. A large series of WD/DDLS yielded evidence of variable amplification of other genes between MDM2 and CDK4, such as HMGA2, YEATS4, and CHOP/ DDIT3.59 Perhaps reflecting a somewhat secondary role of the gene in comparison to MDM2 in this diagnosis, $C D K 4$ is not necessarily amplified in all WD/DDLS. Those that do not have CDK4 amplification are less often found in a retroperitoneal primary location. Perhaps CDK4 amplification can be replaced by other genetic events that provide the same survival advantage in these cases. As a result, we believe that MDM2 and CDK4 are the cores of two distinct amplicons on chromosome 12q.

Other genes on chromosome $12 \mathrm{q}$ also bear further comment. Portions of the HMGA2 gene are consistently amplified, rearranged, and overexpressed in WD/DDLS at 12q14.3. In particular, exons 1 and 2 appear to be the minimal portion of the gene amplified when comparing multiple different copies of the gene from different tumors. It is also characteristically overexpressed in lipomas, as an indicator of the potential importance of the gene in the initiation of adipose tumors. YEATS4 (also called GAS41) interferes with p53 function (as does MDM2), and is amplified in gliomas as well as in approximately $80 \%$ of WD/DDLS. The gene DDIT3 (CHOP), the translocation partner for FUS in myxoid liposarcomas, is often overexpressed in WD/DDLS independent of any amplification, suggesting the importance of this gene in the generation of liposarcomas in general.

Strategies to overexpress these genes in cell lines, as well as knock-down experiments in existing liposarcoma lines, will no doubt tell us more about how these genes function in the future. Clearly, the existence of pharmacological agents that block MDM2 or CDK4 function point to novel agents worth examining in WD/DDLS. Furthermore, preclinical studies using WD/ DDLS cell lines show indications of activity, based on data from cell lines and xenografts. ${ }^{60}$ Hopefully pathology analyses of liposarcoma patient tissues on such therapies will help inform us as to the utility of drugs that block MDM2 or CDK4 function in patients in the near future, since these agents are now available in phase I clinical trials. 


\section{TARGETING ANGIOGENESIS IN SARCOMA}

Blood vessel formation is the first event in embryogenesis that allows for growth, through the development of conduits that carry oxygen and nutrients to developing peripheral tissues. Through a balletic interplay of growth factors and blood vessel precursors, blood vessels are generated during development, typically termed "vasculogenesis." The term "angiogenesis" is generally used for the formation of thin wall endothelial structures with or without smooth muscle and pericytes once initial embryogenesis is complete. Angiogenesis is also the repair mechanism by which blood vessels grow into normal tissues after injury. Arteriogenesis, the development of stronger structures covered with a tunica media and adventitia, is a separate process employing different precursor cells.

There are two forms of angiogenesis, specifically sprouting and splitting/intussiceptive angiogenesis, which are being modeled with increasing sophistication. ${ }^{61}$ The former process involves the use of a gradient of a vascular growth factor to form a leading "bud" of a new vessel into tissue. Splitting angiogenesis refers to the duplication of the cells in the existing (cylindrical) wall of the vessel to make it longer or to allow formation of new branches. These same processes are co-opted by a tumor to allow for neoangiogenesis into tumors for their continued growth.

More than 35 years ago, Judah Folkman, who just recently passed away, proposed that tumors rely on angiogenesis for their continued growth, and that approaches to block this cascade of events would be effective antineoplastic agents. ${ }^{62}$ This was first demonstrated clinically in giant hemangiomas in children with the use of alfa-interferon, although the mechanism remained unclear. ${ }^{63}$ An explosion of research has identified a palette of circulating endothelial factors that are responsible for different aspects of the angiogenic process, including vascular endothelial growth factor (VEGF), fibroblast growth factor 2 (FGF2), transforming growth factor-beta (TGF- $\beta$ ), PDGF, angiopoietins 1 and 2 , angiostatin, endostatin, and others. The function of each of these factors in vasculogenesis, angiogenesis, or arteriogenesis is indicated in Table $2 .{ }^{64}$

Studies in vitro and in vivo have taught us much about the importance of VEGF, FGF2, and other factors in angiogenesis. However, the profound defects of mice made deficient for the genes producing one or another of these factors provide very clear definitions of the functions of these agents. For example, VEGF heterozygous mice are embryonic lethal and have profoundly impaired vasculogenesis. ${ }^{65}$ Conversely, mice completely deficient in FGF2 have intact vasculogenesis but decreased tone in vascular smooth muscle. ${ }^{66,67}$ $P D G F B$ knockout mice lack microvascular pericytes, and develop capillary aneurysm, endothelial hyperplasia, and hemorrhages. ${ }^{68}$

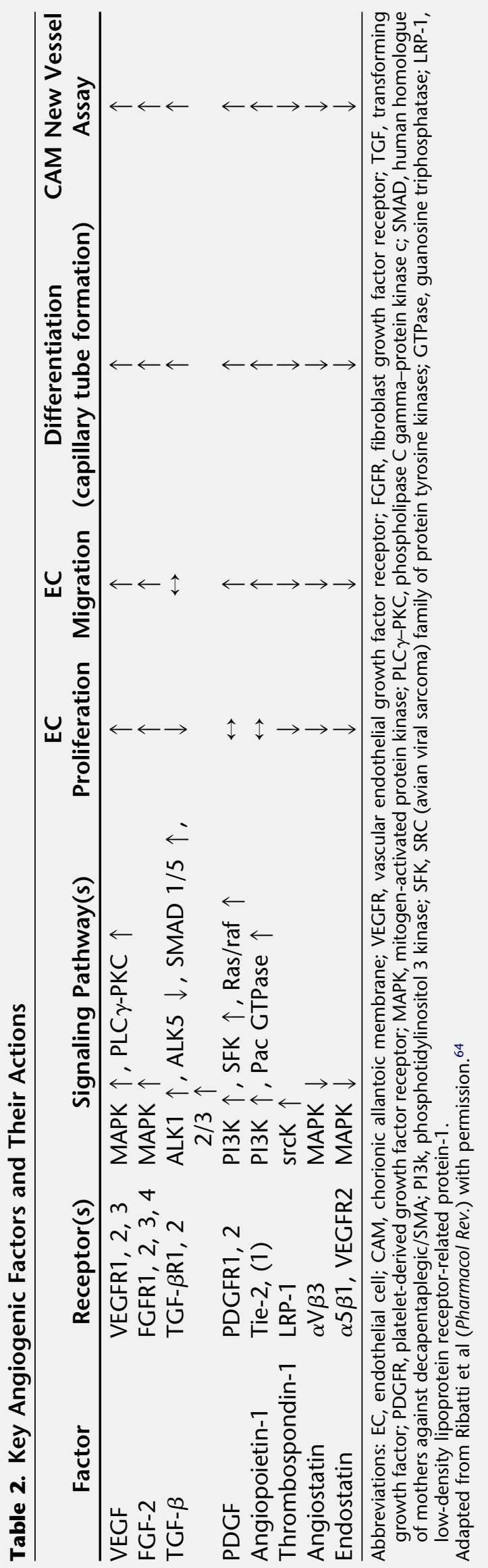


Other sources of information on key genes in angiogenesis include patients with familial syndromes involving vascular abnormalities. For example, hereditary hemorrhagic telangiectasia is characterized by arteriovenous malformations without intervening capillaries, which are caused by defects in TGF- $\beta$ signaling from mutation or deletion of genes such as endoglin (ENG) or activity receptor-like kinase 1 (ACVRL1 = ALK1). ${ }^{69,70}$ Cavernous malformations of the central nervous system also occur in familial syndromes, and involve loss of genes such as CCM1 or mutations in KRIT1. These findings can be recapitulated in mouse models, although the correspondence between the human gene alteration and the mouse gene knockout model is not perfect.

After a slow start in finding agents that block angiogenesis that may be relevant for blocking tumor neoangiogenesis, there is now a plethora of agents, both monoclonal antibody bevacizumab and oral agents, that block signaling of angiogenic pathways in tumors and normal cells alike. One of the first of these was fumagillin analog TNP-470, which inhibits FGF2-mediated endothelial cell proliferation, and showed some activity in early clinical trials, ${ }^{71}$ but ultimately was not approved for human use. ${ }^{71}$

Phase I and II studies of thrombospondin mimetic ABT-510 have shown significant (6 month) disease stabilization for $34 \%$ of patients on study. ${ }^{72}$ Notably, it also was observed that low patient circulating endothelial cell levels in the blood were associated with better prognosis than patients with greater numbers of circulating endothelial cells.

Adding bevacizumab to cytotoxic chemotherapy has improved survival in phase III studies in a variety of epithelial cancers. The combination doxorubicin-bevacizumab was evaluated in a small phase II study of 17 evaluable patients, most with leiomyosarcoma. Only two PRs were observed, which was not enough to merit continuation of the study. ${ }^{73}$ Six patients developed cardiac G2 or worse toxicity on therapy. While some of the toxicity could be attributed to patients receiving up to $600 \mathrm{mg} / \mathrm{m}^{2}$ doxorubicin, four patients developed toxicity with relatively modest cumulative doses of doxorubicin, indicating possible negative effects of bevacizumab when given with other drugs causing potential cardiac toxicity. This small study cannot be considered an adequate evaluation of bevacizumab in patients with sarcoma. For example, a phase II study of bevacizumab against angiosarcoma is underway, but the results are not yet available.

Among the anti-angiogenic TK inhibitors, sunitinib, pazopanib, and sorafenib have all undergone initial evaluation in patients with sarcoma. Sunitinib yielded only PRs in patients with desmoplastic small round cell tumor and minor responses in metastatic hemangiopericytoma/solitary fibrous tumor and metastatic giant cell tumor of bone. ${ }^{74}$ While case reports indicated ac- tivity in chordomas, there were no PRs according to Response Evaluation Criteria in Solid Tumors (RECIST) seen in seven patients treated on the study. ${ }^{74}$

Pazopanib, another inhibitor of VEGF receptors 1, 2, and 3, KIT, and PDGF receptors $A$ and $B$, was examined in a Simon two-stage design in four strata (leiomyosarcoma, liposarcoma, synovial sarcoma, and other sarcomas), with only the liposarcoma stratum not completing accrual due to low rate of PFS at 12 weeks. ${ }^{75}$ Pazopanib is now under study in phase III trials, including one in patients with sarcoma.

Sorafenib was examined in a multicenter study in 147 patients on six different arms. ${ }^{76}$ The only activity observed occurred in $1 / 37$ patients with leiomyosarcoma with a response, and 5/37 angiosarcoma patients with a PR, one of which became a prolonged CR. It is somewhat surprising that more activity was not observed in the angiosarcoma arm, since it is posited that this, of all tumors, should be dependent on VEGF for survival. Minor responses were seen in patients with malignant peripheral nerve sheath tumor and synovial sarcoma, although one patient with synovial sarcoma experienced life-threatening hemoptysis from lung metastases within 10 days of starting sorafenib. This patient developed a PR off protocol when put on a lower dose of sorafenib. Reductions from the US Food and Drug Administration-approved dose of $400 \mathrm{mg}$ oral twice daily were necessary in $61 \%$ of patients due more for skin toxicity than for other adverse events.

While anthracyclines and taxanes have well-recognized activity against angiosarcoma, ${ }^{77-80}$ it also is worth noting that sirolimus has activity against Kaposi sarcoma,${ }^{81}$ which itself is caused by infection with human herpes virus 8 (Kaposi sarcoma herpes virus) ${ }^{82}$ either alone or in concert with human immunodeficiency virus, on the basis of a viral G-protein-coupled receptor that activates TSC2 and mTOR. This gives another line of therapy to consider in combination for patients who have different forms of vascular sarcomas apart from angiosarcoma.

Thus, there appears to be only modest activity of anti-angiogenic therapy against sarcomas of soft tissue and bone; however, combinations of agents are just now being evaluated. Both translational and further clinical trials will better define this important series of signaling pathways in tumors and tumor vasculature in the coming years.

\section{SUMMARY}

The reports presented here indicate a new and exciting phase for the development of molecularly targeted therapeutics in sarcoma. GIST remains the flagship, leading us past the initial excitement associated with proof that rationally designed therapeutic strategies can have an impact in the clinic, so that we now are forced to ask questions as to how to translate 
clinical responses into cures. We are now seeing the extension of the GIST paradigm-target discovery followed by clinical intervention resulting in clinical benefit-to other sarcoma types. These include the role of CSF1 in tenosynovial giant cell tumor, and hopefully MDM2 and CDK4 in well-differentiated and dedifferentiated liposarcoma. While target discovery has traditionally been based on genetic approaches, novel proteomic tools will allow high-throughput identification of pathways that may be targeted in sarcoma which do not carry the mutational "mark of the beast." These non-mutational targets present attractive options for our available armamentarium in diseases like chordoma and ASPS. In other cases, the target is a physiologic process-angiogenesis- even where no direct mutations exist to date. In particular, sarcomas arising in relation to vasculature structures, like angiosarcoma, may be susceptible to anti-angiogenic strategies. Finally, in some cases, the clear sensitivity of some sarcomas to agents such as trabectedin suggests a molecular specificity that remains to be elucidated. The pace of progress is accelerating, and promises much as we move beyond GIST.

\section{Disclosures}

Robert G. Maki: Research support (Pfizer); honoraria (Ziopharm); testimony (Roche)

Margaret von Mebren: Research support (Novartis, Pfizer, Johnson \& Johnson); consultation fees (Novartis, Pfizer, Medimmune)

David Thomas: Research support (Novartis, Amgen, Pfizer)

\section{REFERENCES}

1. Hirota S, Isozaki K, Moriyama Y, et al. Gain-of-function mutations of c-kit in human gastrointestinal stromal tumors. Science. 1998;279:577-80.

2. Grosso F, Jones RL, Demetri GD, et al. Efficacy of trabectedin (ecteinascidin-743) in advanced pretreated myxoid liposarcomas: a retrospective study. Lancet Oncol. 2007; 8:595-602.

3. Schöffski P, Casali PG, Taron M, et al. DNA repair functionality modulates the clinical outcome of patients with advanced sarcoma treated with trabectedin (ET-743) [abstract]. J Clin Oncol. 2006;24:9522.

4. Espinosa I, Lee CH, Kim MK, et al. A novel monoclonal antibody against DOG1 is a sensitive and specific marker for gastrointestinal stromal tumors. Am J Surg Pathol. 2008;32:210-8.

5. Liegl B, Hornick JL, Corless CL, et al. Monoclonal antibody DOG1.1 shows higher sensitivity than KIT in the diagnosis of gastrointestinal stromal tumors, including unusual subtypes. Am J Surg Pathol. 2009;33:437- 46.

6. West RB, Corless CL, Chen X, et al. The novel marker, DOG1, is expressed ubiquitously in gastrointestinal stromal tumors irrespective of KIT or PDGFRA mutation status. Am J Pathol. 2004;165:107-13.

7. Terry J, Saito T, Subramanian S, et al. TLE1 as a diagnostic immunohistochemical marker for synovial sarcoma emerging from gene expression profiling studies. Am J Surg Pathol. 2007;31:240 - 6

8. Dhanasekaran SM, Dash A, Yu J, et al. Molecular profiling of human prostate tissues: insights into gene expression patterns of prostate development during puberty. FASEB J. 2005;19:243-5.

9. Higgins JP, Kaygusuz G, Wang L, et al. Placental S100 (S100P) and GATA3: markers for transitional epithelium and urothelial carcinoma discovered by complementary DNA microarray. Am J Surg Pathol. 2007;31:673-80.

10. Yao R, Lopez-Beltran A, Maclennan GT, et al. Expression of $\$ 100$ protein family members in the pathogenesis of bladder tumors. Anticancer Res. 2007;27:3051-8.

11. Dabbs DJ, Chivukula M, Carter G, et al. Basal phenotype of ductal carcinoma in situ: recognition and immunohistologic profile. Mod Pathol. 2006;19:1506-11.

12. West RB, Rubin BP, Miller MA, et al. A landscape effect in tenosynovial giant-cell tumor from activation of CSF1 expression by a translocation in a minority of tumor cells. Proc Natl Acad Sci U S A. 2006;103:690-5.

13. Blay JY, El Sayadi H, Thiesse P, et al. Complete response to imatinib in relapsing pigmented villonodular synovitis/tenosynovial giant cell tumor (PVNS/TGCT). Ann Oncol. 2008;19:821-2.

14. Tamimi RM, Brugge JS, Freedman ML, et al. Circulating colony stimulating factor- 1 and breast cancer risk. Cancer Res. 2008;68:18-21.

15. Kovacheva VP, Davison JM, Mellott TJ, et al. Raising gestational choline intake alters gene expression in DMBA-evoked mammary tumors and prolongs survival. FASEB J. 2009;23:1054-63.

16. Beck AH, Espinosa I, Gilks CB, et al. The fibromatosis signature defines a robust stromal response in breast carcinoma. Lab Invest. 2008;88:591-601.

17. Cheang MC, van de Rijn M, Nielsen TO. Gene expression profiling of breast cancer. Annu Rev Pathol. 2008; 3:67-97.

18. West RB, Nuyten DS, Subramanian S, et al. Determination of stromal signatures in breast carcinoma. PLoS Biol 2005;3:e187.

19. Lee CH, Espinosa I, Vrijaldenhoven S, et al. Prognostic significance of macrophage infiltration in leiomyosarcomas. Clin Cancer Res. 2008;14:1423-30.

20. Gygi SP, Rochon Y, Franza BR, et al. Correlation between protein and mRNA abundance in yeast. Mol Cell Biol 1999;19:1720-30.

21. Lu P, Vogel C, Wang R, et al. Absolute protein expression profiling estimates the relative contributions of transcriptional and translational regulation. Nat Biotechnol. 2007; 25:117-24.

22. Aebersold R, Mann M. Mass spectrometry-based proteomics. Nature. 2003;422:198-207.

23. Yates JR 3rd, Gilchrist A, Howell KE, et al. Proteomics of organelles and large cellular structures. Nat Rev Mol Cell Biol. 2005;6:702-14.

24. Ong SE, Mann M. Mass spectrometry-based proteomics turns quantitative. Nat Chem Biol. 2005;1:252-62.

25. Cox J, Mann M. Is proteomics the new genomics? Cell. 2007;130:395-8.

26. Izbicka E, Campos D, Marty J, et al. Molecular determinants of differential sensitivity to docetaxel and pacli- 
taxel in human pediatric cancer models. Anticancer Res. 2006;26:1983-8.

27. Gonzalez I, Andreu EJ, Panizo A, et al. Imatinib inhibits proliferation of Ewing tumor cells mediated by the stem cell factor/KIT receptor pathway, and sensitizes cells to vincristine and doxorubicin-induced apoptosis. Clin Cancer Res. 2004;10:751-61.

28. Pollak MN, Schernhammer ES, Hankinson SE. Insulin-like growth factors and neoplasia. Nat Rev Cancer. 2004; 4 : 505-18.

29. Mateo-Lozano S, Tirado OM, Notario V. Rapamycin induces the fusion-type independent downregulation of the EWS/FLI-1 proteins and inhibits Ewing's sarcoma cell proliferation. Oncogene. 2003;22:9282-7.

30. Scotlandi K, Benini S, Sarti M, et al. Insulin-like growth factor I receptor-mediated circuit in Ewing's sarcoma/ peripheral neuroectodermal tumor: a possible therapeutic target. Cancer Res. 1996;56:4570-4.

31. Scotlandi K, Manara MC, Nicoletti G, et al. Antitumor activity of the insulin-like growth factor-I receptor kinase inhibitor NVP-AEW541 in musculoskeletal tumors. Cancer Res. 2005;65:3868-76.

32. Martins AS, Mackintosh C, Martin DH, et al. Insulin-like growth factor I receptor pathway inhibition by ADW742, alone or in combination with imatinib, doxorubicin, or vincristine, is a novel therapeutic approach in Ewing tumor. Clin Cancer Res. 2006;12:3532-40.

33. Martins AS, Ordonez JL, Garcia-Sanchez A, et al. A pivotal role for heat shock protein 90 in Ewing sarcoma resistance to anti-insulin-like growth factor 1 receptor treatment: in vitro and in vivo study. Cancer Res. 2008;68: $6260-70$.

34. Kovar H, Aryee D, Zoubek A. The Ewing family of tumors and the search for the Achilles' heel. Curr Opin Oncol. 1999; 11:275-84.

35. Cohen $P$. The regulation of protein function by multisite phosphorylation-a 25 year update. Trends Biochem Sci. 2000;25:596-601.

36. Armistead PM, Salganick J, Roh JS, et al. Expression of receptor tyrosine kinases and apoptotic molecules in rhabdomyosarcoma: correlation with overall survival in 105 patients. Cancer. 2007;110:2293-303.

37. Casali PG, Messina A, Stacchiotti S, et al. Imatinib mesylate in chordoma. Cancer. 2004;101:2086-97.

38. Lagonigro MS, Tamborini E, Negri T, et al. PDGFRalpha, PDGFRbeta and KIT expression/activation in conventional chondrosarcoma. J Pathol. 2006;208:615-23.

39. Li Y, Chang Q, Rubin BP, et al. Insulin receptor activation in solitary fibrous tumours. J Pathol. 2007;211:550-4.

40. Liegl B, Gully C, Reich O, et al. Expression of plateletderived growth factor receptor in low-grade endometrial stromal sarcomas in the absence of activating mutations. Histopathology. 2007;50:448-52.

41. McArthur GA, Demetri GD, van Oosterom A, et al. Molecular and clinical analysis of locally advanced dermatofibrosarcoma protuberans treated with imatinib: Imatinib Target Exploration Consortium Study B2225. J Clin Oncol. 2005;23:866-73.

42. Rubin BP, Schuetze SM, Eary JF, et al. Molecular targeting of platelet-derived growth factor B by imatinib mesylate in a patient with metastatic dermatofibrosarcoma protuberans. J Clin Oncol. 2002;20:3586-91.
43. Signoroni S, Frattini M, Negri T, et al. Cyclooxygenase-2 and platelet-derived growth factor receptors as potential targets in treating aggressive fibromatosis. Clin Cancer Res. 2007;13:5034-40.

44. Tamborini E, Bonadiman L, Albertini V, et al. Re: potential use of imatinib in Ewing's sarcoma: evidence for in vitro and in vivo activity [letter]. J Natl Cancer Inst. 2003;95:1087-8.

45. Tamborini E, Bonadiman L, Greco A, et al. Expression of ligand-activated KIT and platelet-derived growth factor receptor beta tyrosine kinase receptors in synovial sarcoma. Clin Cancer Res. 2004;10:938-43.

46. Tamborini E, Casieri P, Miselli F, et al. Analysis of potential receptor tyrosine kinase targets in intimal and mural sarcomas. J Pathol. 2007;212:227-35.

47. Tamborini E, Miselli F, Negri T, et al. Molecular and biochemical analyses of platelet-derived growth factor receptor (PDGFR) B, PDGFRA, and KIT receptors in chordomas. Clin Cancer Res. 2006;12:6920 - 8.

48. Heinrich MC, Joensuu H, Demetri GD, et al. Phase II, open-label study evaluating the activity of imatinib in treating life-threatening malignancies known to be associated with imatinib-sensitive tyrosine kinases. Clin Cancer Res. 2008; 14:2717-25.

49. Maki RG, Awan RA, Dixon RH, et al. Differential sensitivity to imatinib of 2 patients with metastatic sarcoma arising from dermatofibrosarcoma protuberans. Int $\mathrm{J}$ Cancer. 2002;100:623-6.

50. Shaw RJ, Cantley LC. Ras, PI(3)K and mTOR signalling controls tumour cell growth. Nature. 2006;441:424-30.

51. Azizi AA, Haberler C, Czech T, et al. Vascular-endothelialgrowth-factor (VEGF) expression and possible response to angiogenesis inhibitor bevacizumab in metastatic alveolar soft part sarcoma. Lancet Oncol. 2006;7:521-3.

52. Lazar AJ, Das $\mathrm{P}$, Tuvin D, et al. Angiogenesis-promoting gene patterns in alveolar soft part sarcoma. Clin Cancer Res. 2007;13:7314-21.

53. Tsuda M, Davis IJ, Argani P, et al. TFE3 fusions activate MET signaling by transcriptional up-regulation, defining another class of tumors as candidates for therapeutic MET inhibition. Cancer Res. 2007;67:919-29.

54. Sirvent N, Coindre JM, Maire G, et al. Detection of MDM2-CDK 4 amplification by fluorescence in situ hybridization in 200 paraffin-embedded tumor samples: utility in diagnosing adipocytic lesions and comparison with immunohistochemistry and real-time PCR. Am J Surg Pathol. 2007;31:1476-89.

55. Italiano A, Cardot N, Dupre F, et al. Gains and complex rearrangements of the 12q13-15 chromosomal region in ordinary lipomas: the "missing link" between lipomas and liposarcomas? Int J Cancer. 2007;121:308-15.

56. Mandahl N, Akerman M, Aman P, et al. Duplication of chromosome segment $12 \mathrm{q} 15-24$ is associated with atypical lipomatous tumors: a report of the CHAMP collaborative study group. CHromosomes And MorPhology. Int J Cancer. 1996;67:632-5.

57. Mandahl N, Heim S, Johansson B, et al. Lipomas have characteristic structural chromosomal rearrangements of 12q13-q14. Int J Cancer. 1987;39:685-8.

58. Storlazzi CT, Mertens F, Domanski H, et al. Ring chromosomes and low-grade gene amplification in an atypi- 
cal lipomatous tumor with minimal nuclear atypia. Int $\mathrm{J}$ Oncol. 2003;23:67-71.

59. Italiano A, Bianchini L, Keslair F, et al. HMGA2 is the partner of MDM2 in well-differentiated and dedifferentiated liposarcomas whereas CDK4 belongs to a distinct inconsistent amplicon. Int J Cancer. 2008;122:2233-41.

60. Ambrosini G, Sambol EB, Carvajal D, et al. Mouse double minute antagonist Nutlin-3a enhances chemotherapy-induced apoptosis in cancer cells with mutant p 53 by activating E2F1. Oncogene. 2007;26:3473-81.

61. Merks RM, Perryn ED, Shirinifard A, et al. Contact-inhibited chemotaxis in de novo and sprouting blood-vessel growth. PLoS Comput Biol. 2008; 4 :e1000163.

62. Folkman J. Tumor angiogenesis: therapeutic implications. N Engl J Med. 1971;285:1182-6.

63. Folkman J. Successful treatment of an angiogenic disease. N Engl J Med. 1989;320:1211-2.

64. Ribatti D, Conconi MT, Nussdorfer GG. Nonclassic endogenous novel [corrected] regulators of angiogenesis. Pharmacol Rev. 2007;59:185-205.

65. Carmeliet P, Ferreira V, Breier G, et al. Abnormal blood vessel development and lethality in embryos lacking a single VEGF allele. Nature. 1996;380:435-9.

66. Tobe T, Ortega S, Luna JD, et al. Targeted disruption of the FGF2 gene does not prevent choroidal neovascularization in a murine model. Am J Pathol. 1998;153:1641-6.

67. Zhou M, Sutliff RL, Paul RJ, et al. Fibroblast growth factor 2 control of vascular tone. Nat Med. 1998;4:201-7.

68. Lindahl P, Johansson BR, Leveen $\mathrm{P}$, et al. Pericyte loss and microaneurysm formation in PDGF-B-deficient mice. Science. 1997;277:242-5.

69. Urness LD, Sorensen LK, Li DY. Arteriovenous malformations in mice lacking activin receptor-like kinase-1. Nat Genet. 2000;26:328-31.

70. Whitehead KJ, Plummer NW, Adams JA, et al. Ccm1 is required for arterial morphogenesis: implications for the etiology of human cavernous malformations. Development. 2004;131:1437-48.

71. Dezube BJ, Von Roenn JH, Holden-Wiltse J, et al. Fumagillin analog in the treatment of Kaposi's sarcoma: a phase I AIDS Clinical Trial Group study. AIDS Clinical Trial Group No. 215 Team. J Clin Oncol. 1998;16:1444-9.
72. Baker LH, Rowinsky EK, Mendelson D, et al. Randomized, phase II study of the thrombospondin-1-mimetic angiogenesis inhibitor ABT-510 in patients with advanced soft tissue sarcoma. J Clin Oncol. 2008;26:5583-8.

73. D'Adamo DR, Anderson SE, Albritton K, et al. Phase II study of doxorubicin and bevacizumab for patients with metastatic soft-tissue sarcomas. J Clin Oncol. 2005;23:7135-42.

74. Keohan ML, Morgan JA, D'Adamo DR, et al. Continuous daily dosing of sunitinib in patients with metastatic soft tissue sarcomas other than GIST: results of a phase II trial [abstract]. J Clin Oncol. 2008;26:10533.

75. Sleijfer S, Papai Z, Le Cesne A, et al. Phase II study of pazopanib (GW786034) in patients with relapsed or refractory soft tissue sarcoma: EORTC 62043 [abstract]. J Clin Oncol. 2007;25:10031.

76. D'Adamo DR, Keohan M, Schuetze S, et al. Clinical results of a phase II study of sorafenib in patients (pts) with non-GIST sarcomas (CTEP study \#7060) [abstract]. J Clin Oncol. 2007;25:10001.

77. Fata F, O'Reilly E, Ilson D, et al. Paclitaxel in the treatment of patients with angiosarcoma of the scalp or face. Cancer. 1999;86:2034-7.

78. Skubitz KM, Haddad PA. Paclitaxel and pegylated-liposomal doxorubicin are both active in angiosarcoma. Cancer. 2005;104:361-6.

79. Penel N, Bui BN, Bay JO, et al. Phase II trial of weekly paclitaxel for unresectable angiosarcoma: the ANGIOTAX study. J Clin Oncol. 2008;26:5269-74.

80. Fury MG, Antonescu CR, Van Zee KJ, et al. A 14-year retrospective review of angiosarcoma: clinical characteristics, prognostic factors, and treatment outcomes with surgery and chemotherapy. Cancer J. 2005;11:241-7.

81. Weenink JJ, Groeneveld JO, de Fijter CW. Sirolimus monotherapy for Kaposi's sarcoma in an HIV-negative patient. Lancet Oncol. 2006;7:875-6.

82. Sodhi A, Chaisuparat R, Hu J, et al. The TSC2/mTOR pathway drives endothelial cell transformation induced by the Kaposi's sarcoma-associated herpesvirus $G$ protein-coupled receptor. Cancer Cell. 2006;10:133-43.

83. Stacchiotti S, Tamborini E, Marrari A, Brich S, Arisi Rota $\mathrm{S}$, Orsenigo $\mathrm{M}$, et al. Response to sunitinib malate in advanced alveolar soft part sarcoma. Clin Cancer Res. 2009;15:1096-104. 